\title{
Evaluation of the Effectiveness of Intervention for Malaria Control in Rural Areas in China
}

\author{
Gorgui Diouf \\ Department of Epidemiology and Biostatistics, School of Public Health \\ Tongji Medical College of Huazhong University of Science and Technology \\ Wuhan, Hubei 430030, China \\ E-mail: gidiouf@hotmail.com \\ Yihua $\mathrm{Xu}$ \\ Department of Epidemiology and Biostatistics, School of Public Health \\ Tongji Medical College of Huazhong University of Science and Technology \\ Wuhan, Hubei 430030, China \\ Patrick N. Kpanyen \\ Department of Epidemiology and Biostatistics, School of Public Health \\ Tongji Medical College of Huazhong University of Science and Technology \\ Wuhan, Hubei 430030, China \\ E-mail: pkpanyen@yahoo.com \\ Li Cai
}

Wuhan Center for Disease Prevention and Control, Wuhan 430015, China

E-mail: kaka2004-02-28@163.com

Xiumin Gan

Department of Epidemiology and Biostatistics, School of Public Health

Tongji Medical College of Huazhong University of Science and Technology

Wuhan, Hubei 430030, China

E-mail: ganxiumin@hotmail.com

Shaofa Nie (Corresponding author)

Department of Epidemiology and Biostatistics, School of Public Health

Tongji Medical College of Huazhong University of Science and Technology

Wuhan, Hubei 430030, China

Tel: 86-27-8369-3763 E-mail: sf_nie@mails.tjmu.edu.cn

\begin{abstract}
Malaria can be prevented at the individual level by taking personal protections. However, effective preventive action is a result of public health programs that adequately teach preventive measures to the population. This study was carried out in 2007 to evaluate the knowledge on malaria control and prevention and to evaluate the effects of intervention for malaria control in rural community in China.
\end{abstract}


An interventional study followed approximately 1971 randomly selected respondents over a period of four months. According to the level of endemicity of the areas, this particular study was carried out in a rural community. Two surveys were performed for the study in point. Demographic and socioeconomic variables were used as predictor variables in logistic regression analysis.

Different patterns of malaria behavior were found in the closely situated households and at the school level. Gender, age, length of residence time in the areas, and health seeking behaviors was found to be statistically significant predictors of health behaviors $(\mathrm{P}<0.001)$. The likelihood between male and female to admit that malaria is a threat for human health was $96.3 \%$ and $97.1 \%$ respectively, (Odds Ratio $=0.782 ; 95 \%$ Confidence Interval $0.458,1.335$; P-value $=0.368)$. The use of mosquito nets was approximately evenly split between genders $(\mathrm{OR}=0.813$; 95\% C.I.: 0.645, 1.025; $\mathrm{P}=0.080)$.

Public health campaigns through iterative actions should be maintained in the region to strengthen the awareness of the population for malaria prevention and control.

Keywords: Community intervention, Evaluation, Health promotion, Malaria control, Malaria prevention

\section{Introduction}

Malaria is a major parasitic disease in China with prevalence that gradually increases from the north to the south. The southern part of China used to be the hyper and meso-endemic regions where falciparum malaria was widely present $(1,2)$. In some meso- and hypo-endemic areas, vivax malaria was predominant, though falciparum malaria also existed and focal outbreaks often occurred. The epidemic situation in China is largely affected by the increase of national travel and immigration from endemics zones (3) and especially by that in the nearby Southeast countries (4). Although great success has been achieved since the launch of the National Malaria Control Programme in 1955, malaria is still an important public health problem in China $(2,5$, 6). Malaria is considerably re-emerging in many provinces and the actual number of cases is much higher than the reported cases (7). The malaria early warning system is not well established in remote areas and the rapid response capability to malaria epidemic by local organizations is limited. The total number of malaria cases in 2003 increased to 40, 681 which is $15.3 \%$ higher than that in 2002 (8). In 2005, the resurgence of malaria in central China was considerable (6), despite the very significant regional decline in the reported malaria cases and deaths during the 1990s, after two decades of malaria control efforts (9). To alleviate the burden of malaria in this region, significant interventions should be carried out to enhance the population awareness on the disease and its degree of control.

Given the fact that malaria is a community or population level problem, it is appropriate to evaluate programs to combat this problem at the higher aggregate level. As with any wide scale problem, this requires certain properties within a study that enable generalization beyond the immediate study group.

It also requires standard measurement of the main outcomes and efforts on behalf of the research community to make the results of research as understandable to as wide an audience as possible (10). Therefore, understanding the local perceptions and practices of the villagers is of utmost relevance to enhance community's potential to deal with village based malaria interventions. The purpose of this study was to evaluate the effectiveness of health promotion in rural community by adopting intervention measures, to improve awareness and demand of the residents for effective malaria prevention and treatment, and to carry out a model that provides a scientific basis for the future evaluation of intervention.

\section{Materials and Methods}

\subsection{Study population}

The study was conducted in Hubei Province, central China. According to the level of endemicity of the areas, this particular study was carried out in a rural community located in the surrounding of Xiangfan city. The villages were chosen at random and interview conducted with trained personnel. The villages were densely populated and separated into clusters. The surveys were performed in 2007 at a four month interval in those clusters with the highest malaria incidence and where knowledge of malaria prevention and the use of personal protection measures among residents were not well established. The local populations within the areas are often vulnerable and appropriate measures of malaria control at the village level are threatened by low economic status. Weaknesses in the health care delivery system, especially at the village level, have produced instability in malaria control efforts and the resulting incidence of malaria.

A total of 1971 respondents that were more likely to disseminate information on health issues within the communities were interviewed. The study population includes household residents and the school populations. 
The school populations were selected as respondents because they had acquired a high level of education and were likely to get some basic information about diseases prevention and control.

\subsection{Study design}

The surveys were design to gather more information from an interventional study of the population. According to the malaria incidence density distribution in Hubei province, two villages (Zaoyang and Xiangyang) and two primary schools in the rural areas of Xiangfan city were selected for the investigation on point. Two consistent surveys questionnaires before and after the intervention, were self-designed. The contents included general demographic characteristics, malaria basic knowledge and common sense, environment and behavior. The surveys were conducted in the same population and the questionnaires were structured in four main parts to develop; knowledge, attitude and practice of malaria control and prevention. The questionnaires were modified after discussion by malaria experts and pre-experimented for official use. Qualified householders were asked about the surveys and filled out the questionnaire before and after the intervention. The surveys were carried out under the supervision of the malaria experts of the regional malaria Center for Disease Control and Prevention.

\subsection{Intervention}

Considering that, with an adequate time and resources, a strategically designed communication can play an important role in scaling up prevention and control efforts at the individual/household and community level. A baseline survey was first completed. Residents in the study area were trained to conduct programs for education on malaria prevention and control. The programs consisted on carrying out lectures, organizing watching self-made malaria control and prevention missionary films, releasing learning materials, streets promotion consulting, layout tour blackboard, newspaper gallery, as well as face to face to guide the exchange of health promotion intervention. We evaluate data in accordance with the local population awareness and needs on malaria prevention and treatment, and develop promotional materials for the prevention. For broadcast requirements; a local television station broadcast once a week. In all primary schools, in each of the highest level, students watched film projection once a month.

\subsection{Statistical Analysis}

Potential factors related to health behavior and some outcome variables were adjusted. For looking for the trend, the chi-square test of the trend was calculated. The categorical variables were described using the logistic regression test to compare the data and analyze the impact of the intervention. For measure of association between different groups, a bi-variate Odds ratio (OR) was calculated with confidence intervals (CI) of 95\%. Epidata version 3.1 and SPSS version18.0, PASWStatistics18 (SPSS Inc. Chicago, Illinois. U.S.) were used for data analysis.

\subsection{Ethical considerations}

This study was reviewed and approved by the ethical committee of the regional Center for Disease Prevention and Control and the Department of Epidemiology and Health Statistics of Tongji medical college. The participation of this study was voluntary and confidentiality of the information assured both before and after intervention. Consent was obtained from all the respondents who participated in the study.

\section{Results}

A total of 1971 respondents were examined during the survey, which included almost every resident present during the survey period. All respondents dwelled in the rural areas surrounding the xiangfan city. The majority of the respondents before and after the intervention were male $57.71 \%$ and $54.29 \%$ respectively. Most of the respondents are Han-chinese.

A baseline survey showed that knowledge on malaria prevention and control among the respondents, were relatively low. Before the intervention (survey 1), knowledge on how to prevent malaria and how it is spread was $37.14 \%$ and $49.10 \%$ respectively. Illness was not treated on time, only $22.33 \%$ of the respondents knew the onset of the symptoms and $4.76 \%$ seek treatment at the onset of the symptoms. Drug used and the number of days for treatment were not well known, $30.95 \%$ and $46.40 \%$ respectively, while $51.64 \%$ continued medication after the symptoms disappeared. Only $10.69 \%$ paid attention to medication. After the intervention (survey 2), we denoted a substantial increase of the awareness of malaria (Table 1). Up to $74.18 \%$ knew how to prevent malaria and $76.31 \%$ was aware on how malaria is spread.

Although the malaria symptoms are sometimes similar to those of many other infections caused by bacteria, viruses, or parasites at the early stages; $59.87 \%$ of the subjects interviewed were likely to identify them and 
$40.79 \%$ sought treatment on time. Knowledge on drug for treatment, $67.63 \%$ and days for treatment, $66.72 \%$ have also increased significantly $(\mathrm{P}<0.01)$.

Although knowledge about mosquitoes transmits malaria was low, mosquitoes bite prevention was also evenly not well known before the intervention. However, after the intervention, residents, when asked about the best way to prevent malaria, $74.18 \%$ mentioned to avoid mosquito bites by using nets and tents, $15.22 \%$ stated preventive treatment, $1.60 \%$ stated community participation through education, and $0.51 \%$ mentioned indoor residual spraying. Some major misconceptions about malaria prevention and control were also found among the respondents. Traditional beliefs were cited; $5.07 \%$ do not know how to prevent malaria and they think that malaria can be prevented by avoiding drinking unboiled water and eating dirty food or to pray "Buddha" for help.

The percentages of participants viewing personal protective behaviors are very or somewhat effective. For each behavior (possessing and sleeping under mosquito nets, indoors sleeping, seeking medical care and doing the blood test) the majority of individuals felt it was an effective form of protection against malaria. Most of the respondents knew at least one method to prevent mosquito bites. Before the effective intervention, $83.65 \%$ of the respondents had mosquito net, $71.90 \%$ of them sleep under mosquito net and $88.68 \%$ avoid outdoors sleeping. After the intervention each of these behaviors was significantly improved by $91.88 \%, 85.19 \%$ and $94.93 \%$ respectively (Table 2). Before the intervention, 78.52\% stated to seek medical care if suffering from malaria. After the intervention it was increased by $92.90 \%$.

However, in remote rural areas many reasons can become blocking factors for health care improvements. One of the factors is the lower socioeconomic status. Financial access appears to be a significant factor, along with physical access. Some respondents affirmed that they do not seek medical care.

For those rural residents, the surveys showed that, $18 \%$ and $20 \%$, respectively before and after intervention, affirmed do not seek medical care because it was seen as too costly, although this is interrelated with both distance and quality.

Others important factors are the distance to the health care center and the time. The distance from the consumers to the health care provider was a strong determinant of where people sought treatment for malaria. Roughly $12 \%$ and $19 \%$ of the residents, respectively during the first and second survey, stated that the health care center was far, while $22 \%$ and $18 \%$ stated that they do not have time. The perceived quality of services was also an important determinant. More importantly, rural residents access lower quality health services, including greater use of traditional healers, and less likelihood of using public and private hospitals and clinics. Surprisingly, 12\% of the residents considered that it is useless to seek for medication in a health care center if they were suffering from malaria. After the intervention $4 \%$ of them still have the same idea. Additionally, $36 \%$ and $39 \%$ do not seek medication for unknown reasons (Figure 1).

Looking for association between groups, we collected information from the whole population including residents and the school children. Respondents were asked in an open-ended format about their awareness on malaria. Most of those interviewed, $90.65 \%$ of the male and $91.18 \%$ of the female declared they heard about malaria. There is no significant difference between gender (Odds ratio OR $=1.017$; and 95\% Confidence Interval C.I.: $0.723,1.428 ; \mathrm{P}=0.924)$. A least $81.5 \%$ of the male declared that malaria is transmitted by mosquito bites and $83.4 \%$ of the female knew that mosquito transmit the disease.

About $73.37 \%$ of the respondents attested that malaria is a serious harmful infectious disease for human health. However, the likelihood between male $96.3 \%$ and female $97.1 \%$ to admit that malaria is a threat for human health is not significant $(\mathrm{OR}=0.782 ; 95 \%$ C.I.: $0.458,1.335 ; \mathrm{P}=0.368)$. About $10 \%$ of the respondents were immigrants and $78.2 \%$ of them agreed with the statement and $69 \%$ use mosquito nets for protection (Table 3 ).

The vast majority of the population was young. The study reported that $69.4 \%$ was less than $15 y e a r s ; 5.6 \%$ have an age range between $15-30$ years; $22.45 \%$ between $30-50$ years and only $2.5 \%$ were above 50 years. The slight majority of the respondents were school children, and educational level was significantly higher in the youngest group, $86.31 \%$. We found very different patterns of malaria behavior in the closely situated houses. Malaria knowledge, attitude and practices for personal protection vary strongly among the residents as well as the school children. The study showed that, people living in Xiangyang village had better understanding on malaria than those in Zaoyang village. It was also noted that in Xiangyang village, $98.4 \%$ of the school children declared that they have heard about malaria. They are also more likely to admit that malaria is a treat for human health, $98 \%$ $(\mathrm{OR}=0.171 ; 95 \%$ C.I.: $0.119,0.248 ; \mathrm{P}<0.001)$; knew that mosquitoes transmit malaria, $87.1 \%(\mathrm{OR}=0.586$; 95\% C.I.: 0.499, 0.688; $\mathrm{P}<0.001)$; and therefore slept under mosquito net $99.4 \%(\mathrm{OR}=0.015 ; 95 \%$ C.I.: 0.005 , $0.041 ; \mathrm{P}<0.001$ ) (Table 3). 
It was reported that $85.19 \%$ of the respondents use preventive methods such as mosquito nets. The constant use of mosquito nets, was approximately evenly split between male $94.5 \%$ and female $96.3 \%$ (OR $=0.813$; $95 \%$ C.I.: $0.645,1.025 ; \mathrm{P}=0.080$ ).

For those who did not sleep under mosquito net, there were some differences in the perception of the use of the net. It was estimated that $30.57 \%$ did not use net because they did not know that it could prevent malaria, $30.36 \%$ for trouble reasons, $26.58 \%$ for heat intolerance and $12.28 \%$ for others reasons. For evaluating the susceptibility to malaria, the study revealed that respondents consider that those who were engaged in field operations were the more susceptible $73.7 \%$, followed by immigrant from a nonendemic area to an endemic area $(47.3 \%)$ and only $(6.78 \%)$ for those who stay at home.

The concept of health management through community education is important in rural areas. The study showed that $76.26 \%$ of the respondents affirmed that health education to change the undesirable behavior is the best way to promote the prevention of malaria. Participants were asked whether they agreed with the statement that education through lectures, publicity or advertising campaigns were the best way to disseminate information on malaria protection and control (Figure 2). Roughly $60 \%$ of the sample disagreed with the statement with regards to lectures, while the rest stated they either agreed $(27 \%)$, education through lectures was better or they were unsure (13\%). Slightly less than half (48\%) agreed when asked if they believed education through publicity was better and (42\%) agreed with the statement with regards to the advertising campaigns. The remaining half of the population was split between with disagreeing with the statements, or being unsure.

\section{Discussion}

It is particularly important to understand the populations' awareness of malaria control and prevention to implement programs for future interventions in rural areas.

It is also important to identify those groups who are able to disseminate information on malaria prevention to the others in the community. Key informants must be chosen in any survey because they are the information givers in remote rural areas. Many interventions for reducing the burden of malaria and other diseases depend on improved consumers' knowledge about the disease and its control and this is enhanced by increased educational attainment (11). Educational attainment and knowledge of malaria both play a role in peoples' perceptions and practice for controlling malaria (12). This has been proved by similar studies in Tanzania where perceived symptoms was significantly associated with having primary education and above (13), and in Laos where school-based malaria education has been shown to be effective for improving the knowledge, attitudes, and practices of school children toward malaria control (14).

The level of knowledge with regards to disease transmission varies, and studies show that people may not understand that mosquitoes spread a number of diseases. The preventive measures taken by the population is strongly related to the perception and the understanding about the cause and the transmission of malaria.

Through this study, we found that in remote areas, traditional considerations can lead to misconceptions. Residents believe that taking bathe with cold water or drinking unboiled water can cause malaria. The same consideration has been found in Uganda, where a study showed that many people believe that in addition to mosquitoes, drinking dirty water, inhaling bad air, witchcraft, and eating fresh fruit can cause malaria (15). In Ghana, malaria is presumed to be caused as a result of excessive heat and eating oily or starchy food (16). Explanations of the disease were often related to spiritual and hereditary causes (17).

It is very apparent within the community that they do not believe mosquitoes transmit the disease. The only reason any measures are taken against mosquitoes by some individuals (using coils, herbs, or insect repellent) is simply because insects are a nuisance (17).

Additionally, residents in Thailand consider mosquitoes to be an annoyance, but do not perceive disease as a major risk associated with them (18). It is also well recognized that accessibility to anti-malaria interventions alone will not bring about the desired change. Several studies have demonstrated compliance to anti-malaria interventions depends substantially on social, behavioral and cultural factors that affect understanding of the causes, the relationship between mosquitoes and the disease, diagnosis, treatment and practices about prevention $(19,20)$. In addition, factors such as vulnerability, economic constraints, inadequacy or unavailability of appropriate health services, and other related factors play an important role in explaining health seeking behavior of the people (21). To better confront and solve the considerable malaria misconception through out the population in rural areas, an increased number of well-trained and active scientists and institutions should join effort for operational challenges. It is important not only to understand disease systems, but also to develop control strategies to reduce the number of cases in a population. It is therefore crucial that public health messages 
help to educate the population about the health threats in the community. A widespread public information campaign about malaria prevention is a first step in recruitment both because it can attract a representative cohort of participants and because it provides opportunity to educate potential subjects. The development of educational methods that ensure ethical informed consent, likely an iterative process will be facilitated by the results of the studies on willingness to participate. The current lack of accurate documentation and the socioeconomic situation of the residents in the areas insight that scaling up the endemicity of malaria in the region did not receive the attention or resources they deserve. The reason for which residents stated not to seek medical care if suffering from malaria might be due to the weaknesses of the health care services and the lack of information. Equitable improvement of treatment-seeking for malaria will depend on how different socio-economic groups perceive the ease of accessing and utilizing malaria treatment services from different healthcare providers (22). Adapted health care services with qualified personnel workers still are an urgent need. Studies showed that epidemics tend to affect marginalized and underserved population that may be outside of the usual reporting systems (23). In some instances, more cases will go unreported than the usual families may be unable to attend facilities for financial or health reasons, both of which will be compounded in a severe epidemic (23). Although short-term malaria health education campaigns may have a positive impact on the control of malaria (12), a multi-channel approach is required to overcome the weaknesses inherent in individual channels (24). The involvement of politicians and opinion leaders, community advocates and local media in campaign is essential for the design and the conduct of a survey. Communication efforts should be strategically designed from an audience perspective to address the social and contextual environment as well as individual behaviors and knowledge. Integrating strategic communication approaches and service delivery can enhance utilization of services and improve client compliance. In fact, the integration of community based distribution of anti-malarial and malaria information and education has been documented to reduce under-five mortality by $41 \%$ in one Ethiopian programme $(25,26)$.

\section{Conclusion}

This study suggests how perceptions and practices vary within a group of individuals and is of use to public health officials dealing with both mosquito-borne and other types of diseases. Effective advocacy among influential individuals and groups can also help to address some of the underlying societal and environmental factors that influence individuals' ability to take action, either in terms of prevention or treatment.

Within the framework of the Health Belief Model, public health campaigns in the study region can be strengthened by raising awareness of mosquito-borne disease present in the area, by understanding what the general population feels, and what preventative methods are generally accepted. It would be important for public health officials to know if there is a temporal factor related to how long public health messages may be retained in a community. This is another issue that can be specifically addressed by public health campaigns to clear up misconceptions about malaria control and prevention.

\section{Acknowledgments}

The authors thank the staff of the Center for Disease Control and Prevention for their help for data collection, data entry and for the field work. We are indebted to all the respondents participating in this study. The field supervisors, the field workers, data managers were important for the successful completion of the study.

\section{References}

Agyepong IA, Manderson L. (1999). Mosquito avoidance and bed net use in the Greater Accra Region, Ghana. $J$ Biosoc Sci, 31:79-92.

Agyepong IA. (1992). Malaria: ethnomedical perceptions and practice in an Adangbe farming community and implications for control. Soc Sci Med, 35:131-137.

Ahorlu C, Dunyo S, Koram K, et al. (1999). Lymphatic Filariasis related perceptions and practices on the coast of Ghana. Implications for prevention and control. Acta Tropica, 73: 251-261.

Ahorlu CK, Dunyo SK, Afari EA, et al. (1997). Malaria related beliefs and behaviour in southern Ghana: implications for treatment, prevention and control. Trop Med Int Health, 2:488-499.

Breman, J.G. (2001). The ears of the hippopotamus: Manifestations, determinants, and estimates of the malaria burden. American Journal of Tropical Medicine and Hygiene, 64(1, 2) S.

Control of Malaria in East Asia. (2004). Report of the Bi-Regional Meeting Shanghai, People's Republic of China, 29 November - 2 December.

Dike N, Onwujekwe O, Ojukwu J, et al. (2006). Influence of education and knowledge on perceptions and practices to control malaria in Southeast Nigeria. Social Science \& Medicine, 63, 103-106. 
Eisele T, Macintyre K, Eckert E, et al. (2000). Evaluating malaria interventions in Africa: A review and assessment of recent research. February.

Hausmann-Muela S, Ribera JM, Nyamongo I. (2003). Health Seeking Behaviour and the Health System Response DCPP Working Paper No. 14.

Li HF, Xu BZ, Webber R. (1995). Primary health care: the basis for malaria control in Hubei, China. Southeast Asian Journal of Tropical Medicine and Public Heath. Mar: 26(1):29-33.

Lin HL, Lu L, Tian LW, et al. (2009). Spatial and temporal distribution of falciparum malaria in China. Malaria Journal, 8:130 doi:10.1186/1475-2875-8-130.

Marsh V, Kachur SP. (2002). Malaria Home Care and Management, Policy to Strategy and Implementation Series, Malaria Consortium, December.

Miller JP, Garcia de Ollala P, Carrillo-santisteve P, et al. (2008). Imported malaria in a cosmopolitan European city: A mirror image of the world epidemiological situation. Malaria journal, 7:56.

Mitchell K, Nakamanya S, Kamali A, et al. (2001). Community-based HIV/AIDS education in rural Uganda: which channel is most effective? Health Educ Res, 16:411-23.

Na-Bangchang K, Congpuong K. (2007). Current malaria status and distribution of drug resistance in East and Southeast Asia with special focus to Thailand. Tohoku J Exp Med, 211:99-113.

Nonaka D, Kobayashi J, Jimba M, et al. (2008). Malaria education from school to community in Oudomxay province, Lao PDR. Parasitology International, 57, 76-82.

Nuwaha, F. (2001). People's Perception of Malaria in Mbarara, Uganda. Tropical Medicine and International Health, 7 (5): 461-470.

Onwujekwe O, Uzochukwu B, Eze S, et al. (2008). Improving equity in malaria treatment: Relationship of socio-economic status with health seeking as well as with perceptions of ease of using the services of different providers for the treatment of malaria in Nigeria. Malaria Journal, 7:5 doi: 10.1186/1475-2875-7-5.

Phuanukoonnon S, Brough M, Bryan J. (2006). Folk Knowledge about Dengue Mosquitoes and Contributions of Health Belief Model in Dengue Control Promotion in Northeast Thailand. Acta Tropica, 99: 6-14.

Tang LH. (2000). Progress in malaria control in China. Chin Med J, 113:89-92.

Tarimo DS, Lwihula G K, Minjas JN, et al. (2000). Mothers' perceptions and knowledge on childhood malaria in the holoendemic Kibaha district, Tanzania: Implications for malaria control and the IMCI strategy. Tropical Medicine and International Health, 5(3), 179-184.

WHO, UNICEF. (2003). The Africa Malaria Report, Chapter 3: Prompt and effective treatment, pp $31-37$.

Worrall E, Riertveld A, Delacollette C. (2004). The burden of malaria epidemics and cost-effectiveness of interventions in epidemic situations in Africa. Am. J. Trop. Med. Hyg., 71(Suppl 2), pp. 136-140.

Zhang WY, Wang LP, Fang LQ, et al. (2008). Spatial analysis of malaria in Anhui province, China. Malaria journal, 7:206.

Zhong X N. Current status of malaria in China. National Institute of Parasitic Diseases, China CDC.

Zhou SS, Wang Y, Tang LH. (2006). Malaria Situation in the People's Republic of China in 2005. Chin J Parasitol Parasit Dis, Dec, 24(6):401-403. 
Table 1. Malaria-related KAP comparison in the general population before and after the intervention

\begin{tabular}{|c|c|c|c|c|}
\hline \multirow[b]{2}{*}{ Parameter } & \multicolumn{2}{|c|}{ Knowledge, Attitude, and Practice (\%) } & \multirow[t]{2}{*}{$\chi^{2}$} & \multirow[t]{2}{*}{$P$} \\
\hline & survey 1 & survey 2 & & \\
\hline How malaria is spread & 49.10 & 76.31 & 306.32 & $<0.01$ \\
\hline Onset of the symptoms & 22.33 & 59.87 & 560.09 & $<0.01$ \\
\hline Seeking treatment on time & 4.76 & 40.79 & 703.90 & $<0.01$ \\
\hline $\begin{array}{l}\text { What to do if suffering } \\
\text { from malaria }\end{array}$ & 79.79 & 90.11 & 80.81 & $<0.01$ \\
\hline Drug for treatment & 30.90 & 67.63 & 519.21 & $<0.01$ \\
\hline Days for medication & 46.40 & 66.72 & 162.26 & $<0.01$ \\
\hline Attention to medication & 10.69 & 39.37 & 419.68 & $<0.01$ \\
\hline \multicolumn{5}{|l|}{ Continue medication } \\
\hline after symptoms disappeared & 51.64 & 81.18 & 379.08 & $<0.01$ \\
\hline Prevent malaria & 35.19 & 74.18 & 592.85 & $<0.01$ \\
\hline
\end{tabular}

KAP: Knowledge, Attitude, Practice; $\quad \chi^{2} \quad$ chi-square, $P$ : $P$-value. The surveys were performed respectively before and after the intervention. Residents were asked questions to ensure whether they know how malaria is spread, how to prevent, and if they can recognize the onset of the symptoms, and what to do if suffering from malaria. The types of drug used for treatment and medication schedule were also asked.

Table 2. Malaria personal protection and prevention before and after intervention

\begin{tabular}{lcccc}
\hline & \multicolumn{1}{c}{ Personal protection and prevention (\%) } & \multirow{2}{*}{$\chi^{2}$} & \multicolumn{1}{c}{$P$} \\
\cline { 2 - 3 } Parameter & Survey 1 & Survey 2 & & $<0.01$ \\
\hline Have mosquito nets & 83.65 & 91.88 & 61.26 & $<0.01$ \\
Sleep under mosquito net & 71.90 & 85.19 & 101.46 & $<0.01$ \\
Indoors sleeping & 88.68 & 94.93 & 57.10 & \\
Seek medical care after & 78.52 & & & $<0.01$ \\
Suffering from malaria & 84.81 & 91.32 & 124.47 & $<0.01$ \\
Blood test & & 92.90 & 64.05 & \\
\hline
\end{tabular}

Personal protection is widely used and accepted to prevent mosquito bites. The number of respondents was stratified by those who have and use mosquito bed nets, indoor sleeping, seeking medical care for malaria, and accept the blood test for malaria. After the intervention, there is an increase of good practice for personal protection and prevention for malaria, and the trend is statistically significant $(P<0.01)$. 


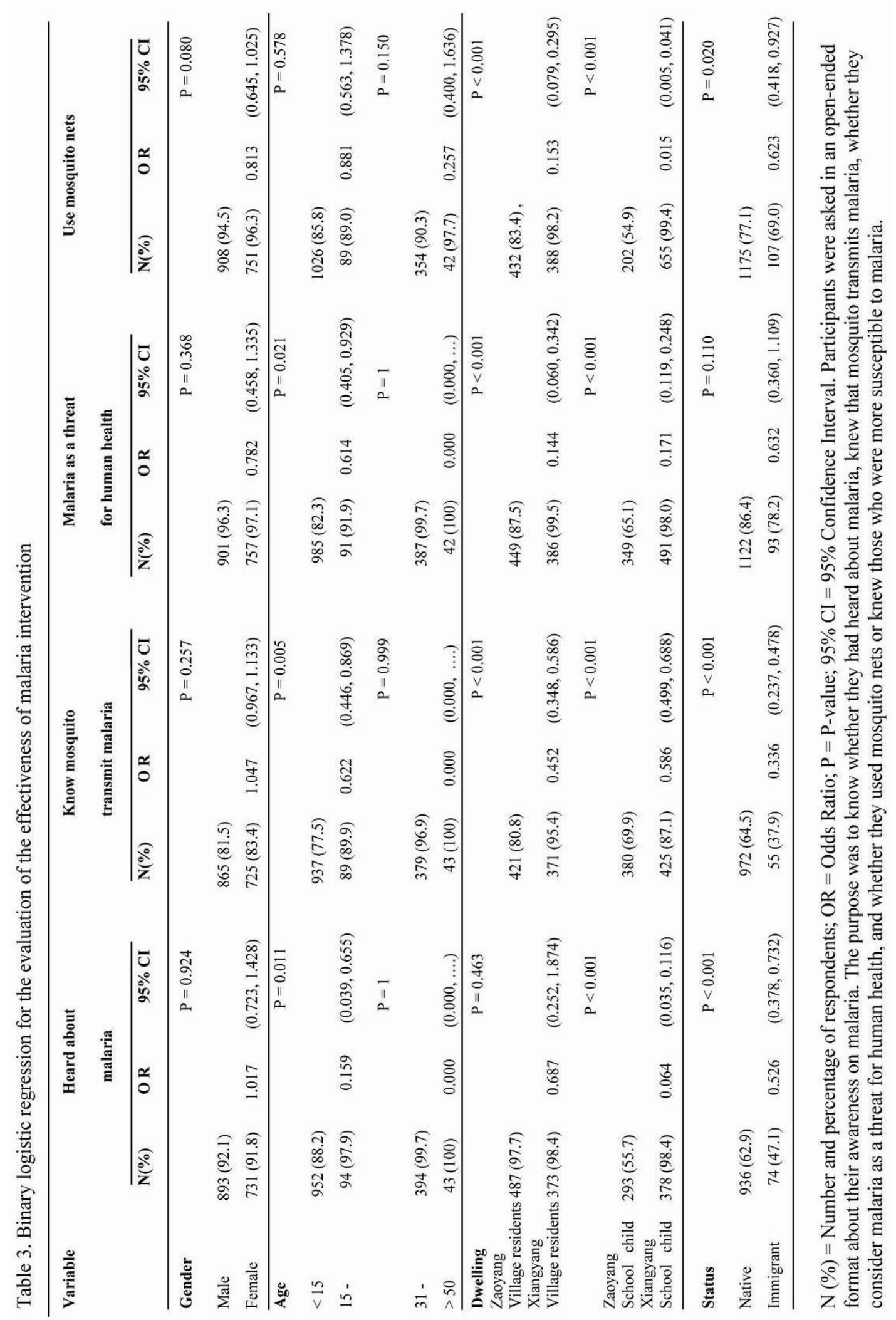




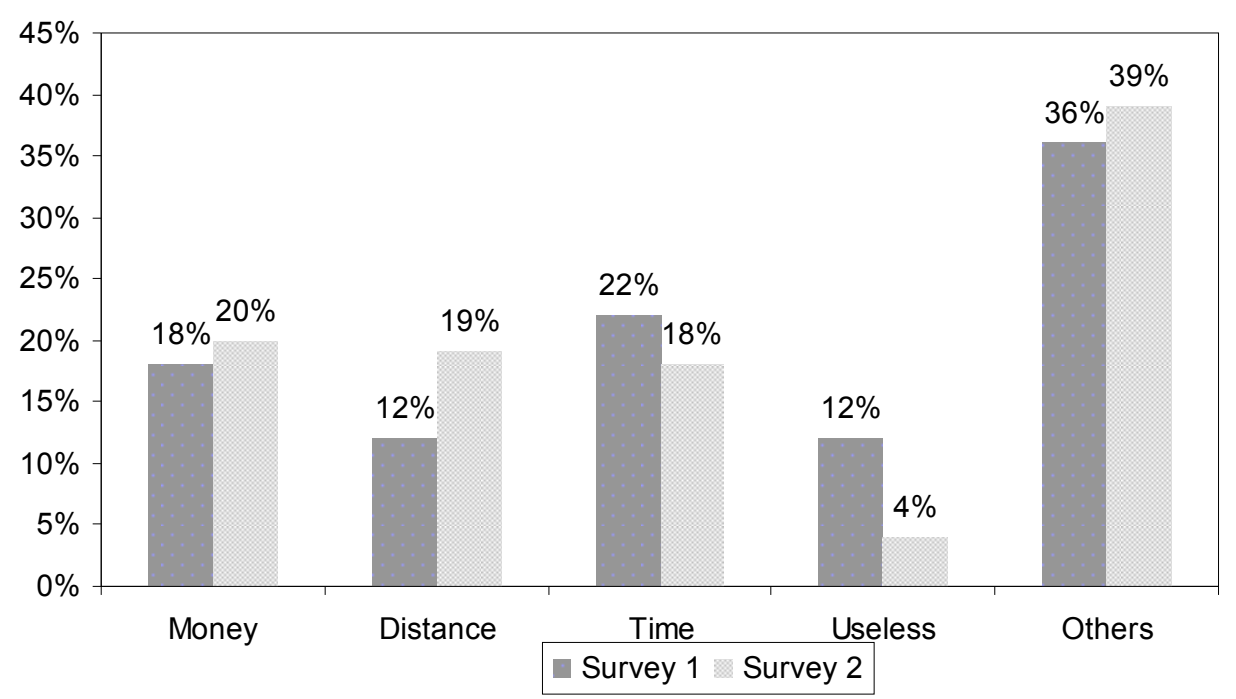

Figure 1. Different reasons as to why respondents do not see a doctor if suffering from malaria

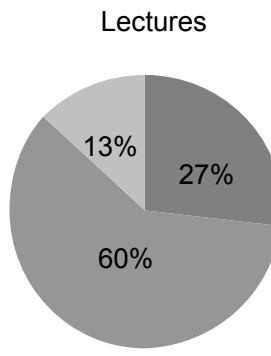

Agree

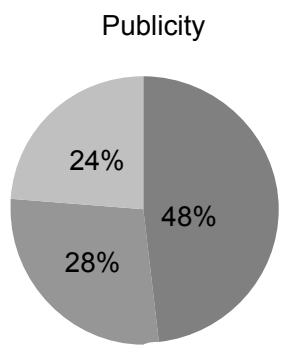

Unsure
Advertising Campaigns

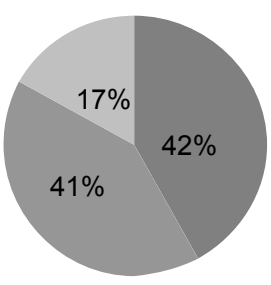

Figure 2. Agreement to the statement that education can disseminate information on malaria control and prevention 